\title{
Assessing the Impact of Automatic vs. Controlled Rotations on Spatial Transfer with a Joystick and a Walking Interface in VR
}

\author{
Florian Larrue ${ }^{1,2}$, Hélène Sauzéon ${ }^{2,1}$, Déborah Foloppe ${ }^{3}$, Grégory Wallet ${ }^{4}$, \\ Jean-René Cazalets ${ }^{5}$, Christian Gross ${ }^{6}$, Martin Hachet ${ }^{1}$, and Bernard N'Kaoua ${ }^{1,2}$ \\ ${ }^{1}$ INRIA, F-33400 Talence, France \\ ${ }^{2}$ University of Bordeaux Victor Segalen - EA 4136, Handicap \& Système Nerveux \\ 33076 Bordeaux Cedex, France \\ ${ }^{3}$ LUNAM Université - Université d'Angers - LPPL - (UPRES EA 4638), France \\ ${ }^{4}$ Aix-Marseille Université, CNRS, ISM UMR 7287, 13288, Marseille Cedex 09, France \\ ${ }^{5}$ CNRS UMR 5287 - INCIA - Institut de Neurosciences Cognitives et Intégratives d'Aquitaine \\ 33076 Bordeaux Cedex, France \\ ${ }^{6}$ Institut des Maladies Neurodégénératives - University of Bordeaux Victor Segalen \\ CNRS UMR 5293 - 33076 Bordeaux Cedex, France \\ \{flo.larrue, gregwallet, foloppe.deborah\} @gmail.com, \\ \{helene.sauzeon, christian.gross, jean-rene.cazalets, \\ bernard.nkaoua\} @u-bordeaux2.fr, \\ martin.hachet@inria.fr
}

\begin{abstract}
We present a user study assessing spatial transfer in a 3D navigation task, with two different motor activities: a minimal (joystick) and an extensive motor activity (walking Interface), with rotations of the viewpoint either controlled by the user, or automatically managed by the system. The task consisted in learning a virtual path of a 3D model of a real city, with either one of these four conditions: Joystick / Treadmill Vs Manual Rotation / Automatic Rotation. We assessed spatial knowledge with six spatial restitution tasks. To assess the interfaces used, we analyzed also the interaction data acquired during the learning path. Our results show that the direct control of rotations has different effects, depending on the motor activity required by the input modality. The quality of spatial representation increases with the Treadmill when rotations are enabled. With the Joystick, controlling the rotations affect spatial representations. We discuss our findings in terms of cognitive, sensorimotor processes and human computer interaction issues.
\end{abstract}

Keywords: Interfaces, Navigation, Virtual Reality, Spatial Cognition, Joystick, Treadmill, Rotation, Body-based Information, Vestibular Information, Human Machine Interaction, Human Factors, User Study, Motor Activity.

\section{Introduction}

Today, Virtual Reality (VR) enables the simulation of dynamic, three-dimensional, multimodal environments. Moreover, this technology allows immerging users in 
different simulations close to real situations, where users can interact with the virtual environment (VE) and have a motor and a cognitive activity. VR also permits the access to various data (e.g. completion time and precision), hard to reach in a real environment. Thanks to these advantages, VR is well suited to create therapeutic applications for patients with spatial disabilities diseases. An important question to explore for such application is to evaluate how spatial information is impacted when transferred from virtual to real environments. Several studies already found great results with disabled people about the question of spatial transfer [25][26][27]. In this work, the authors agree that various factors can enhance this spatial transfer. One question not yet resolved concerns the exploration mode used to navigate in a VE [22]. Indeed, sometimes authors have shown great spatial acquisition with an active exploration mode (i.e., user had a sensorimotor interaction with the VE) compared to a passive mode exploration [23] (i.e., user had no interaction with the VE) [21][1][2][8], but others did not [22][23][24]. Moreover, these studies were generally based on a joystick or a mouse/keyboard interface. Nevertheless, different authors demonstrated, that a walking activity allows to optimize the acquisition of spatial knowledge [12][13], but only two concerns the spatial transfer [3][19]. Thus, we first purpose to assess the impact of two motor activities on spatial transfer with two Input Devices: a walking interface (using a Treadmill) and a Joystick. Moreover, the impact of rotation movements during a navigational activity, on spatial transfer, is not yet clear. In a second step, we therefore investigated the role of Rotation (Automatic-i.e., controlled by the computer- Vs Controlled-i.e., managed by the user) with the two Input Devices presented above. So, on a spatial transfer task, we used i) a Treadmill with Controlled Rotation ii) a Treadmill with Automatic Rotation iii) a Joystick with Automatic Rotation iv) a Joystick with Controlled Rotation. We used six tasks to assess spatial knowledge. To our knowledge, this study is the first one describing impact of translational and directional movements according to different motor activities in a VE, on spatial transfer.

\subsection{Spatial Cognition (cognitive and sensorimotor processes)}

Spatial cognition refers to cognitive and motor processes requiring to acquire, to store and to restitute spatial knowledge. Processes involved in spatial cognition are necessary for many daily life situations, such as shopping in supermarkets (e.g., finding a product in a section) and driving, and are often affected by neurological diseases (e.g., Alzheimer), brain trauma, etc. For Montello [28], spatial cognition is divided in two components: 1) the motor component, composed of all sensorimotor information acquired during a displacement, with visual, kinesthesic and vestibular information, informing on the position and the orientation of the head/body in an environment; 2) the cognitive component corresponding to the processes used to acquire, store and restitute spatial knowledge. One of the most known spatial acquisition model is the Landmark-Route-Survey model of Siegel and White [9]. For this model, spatial knowledge acquisition of new environments consists of three stages. Firstly, spatial cognition is based on the acquisition of several landmarks in the environment. Secondly, the participant links the landmarks and learns the routes between them. At this 
level, s/he is able to build a mental representation of a route from a departure point to an arrival point using the various landmarks. These first two levels correspond to egocentric-type representations (i.e., the body serves as a reference). Finally, the participant develops survey knowledge. S/he builds a topographical representation of the environment, including all the associated spatial information (i.e., landmarks and routes), making it possible to infer a representation of the entire environment, making it possible to contemplate shortcuts. At this final level of knowledge, the representation is similar to a "plane view" and is also known as "survey-type" knowledge: the mental representation of the environment is complete and allocentric (i.e., an external point serves as a reference). These three acquisition stages need not follow a strict order but may be obtained in a parallel process [29]. Concerning the sensorimotor component, body-based information required during a navigational activity can be divided in three types of information [14]: 1) the optic flow, consisting of all visual input used to detect forms, textures, semantic landmarks, movements of objects, etc. always in relation with body position, 2) the vestibular system provides translational (acceleration/deceleration of the head and body) as well as rotational information (rotation of the head and body), and 3) the kinesthetic information, which informs about the perception of our members according to our body. In real environments, different authors admitted that vestibular information is important to the creation of egocentric representations (perception of distances, angles or route knowledge) or to store a direction linked to an egocentric point of view [11] [12], while allocentric representations would be more sensible to visual information.

\subsection{Spatial Cognition, Interfaces and Rotational Movements in VR}

Literature concerning walking activity in VR is not very consistent. However, most of the studies agree that the extent of body-based information provided by a treadmill locomotion interface (compared to a joystick) was considered largely favorable for spatial learning in a VE [11][13][14], due to the improvement in egocentric [11] and allocentric spatial representation [14], as well as navigational measurements [19]. Recently, Ruddle et al. [14] assessed the role of both translational and rotational vestibular information on survey knowledge, using different locomotion interfaces (translational displacements with walking or treadmill Vs. no translational displacements with joystick), sometimes with the possibility of really turning the head (i.e., rotational vestibular condition or not) during rotational movement. Performances revealed an improvement of survey knowledge with a walking activity, but little effect about rotational vestibular information was observed. For Waller et al.[11], the low level of body-based information provided by the design of the locomotion interfaces of the desktop VEs (i.e., keyboards, mouse or joysticks) do not allow the increase of spatial knowledge acquisition. For some authors [16], the manipulation of translational and rotational movements with a joystick demands a strong motor and cognitive attentional levels, which could interfere on spatial learning acquisition. Even if authors promote a walking interface to optimize spatial knowledge, it seems it is still possible to navigate in a VE with a joystick [1][2], without vestibular information[3]. However, in all the studies presented we did not find research, whatever the interfaces used 
(and body-based information provided), where the possibility to perform rotation of the user's viewpoint was disabled and managed automatically by the system.

\subsection{Spatial Cognition and Spatial Transfer from Virtual to Real Environments}

One important challenge of VR is to detect the factors promoting knowledge acquisition in VR to improve daily life activities in the real life. Different authors already showed great spatial transfers with "normal"[1][2][3] or patients with disabilities people [27]. Numerous factors like visual fidelity [2], retention delay [1], game experience [16] increase this transfer. However, concerning the motor activity of the interfaces used, most parts of studies used a passive exploration mode, or a joystick/mouse/keyboard interface (active exploration mode) to navigate in the VE. And the results point out sometimes great performances for the active exploration mode, [21][1][2][8], and others did not [22][23][24]. Moreover, these interfaces don't provide vestibular information, known to improve spatial acquisition. We found only two studies which used a walking interface to study spatial transfer. The first [19] revealed a better spatial transfer with a walking interface compared to a joystick, concluding on the importance of vestibular information. The second study [3] assessed the impact of the motor activity in a spatial transfer task. They compared a Brain Computer Interface (allowing to navigate in a VE with no motor activity), a treadmill interface (enabling vestibular information), and a learning path in the real environment. The results revealed similar performances, whatever the learning conditions, indicating that the cognitive processes are more essential than a motor activity. Results revealed also that a walking activity (and vestibular information) enables spatial knowledge transfer similar to the real life.

\section{$2 \quad$ Method}

VR was assessed as a spatial learning medium using a spatial learning paradigm that involved acquiring a path in its virtual replica [1][2][3]. In our experiment, the acquisition path in the VE was assessed according to four conditions: (1) Treadmill with Controlled (head) Rotation (optic flow, rotational and translational vestibular information); (2) Treadmill with Automatic Rotation (optic flow, translational vestibular information and no rotational vestibular information); (3) Joystick with Controlled (hand) Rotation (optic flow); (4) Joystick with Automatic Rotation (optic flow). Following VR-based path acquisition, the participants completed six tasks to assessing their spatial knowledge and spatial transfer.

\subsection{Setup}

The environment.

The real environment was a $9 \mathrm{~km} 2$ area. The VE was a $3 \mathrm{D}$ scale model of the real environment, with realistic and visual stimuli. The scale of the real environment was faithfully reproduced (measurements of houses, streets, etc.) and photos of several 
building facades were applied to geometric surfaces in the VE. Significant local and global landmarks (e.g., signposts, signs, and urban furniture) and urban sounds were included in the VE (see Figure 1). VE was laboratory-developed using Virtools Dev $3.5^{\mathrm{TM}}$. Irrespective of the interfaces conditions, the itinerary was presented to participants on the basis of an egocentric frame of reference, at head height. It was characterized by an irregular closed loop, $780 \mathrm{~m}$ in length, with thirteen crossroads and eleven directional changes.
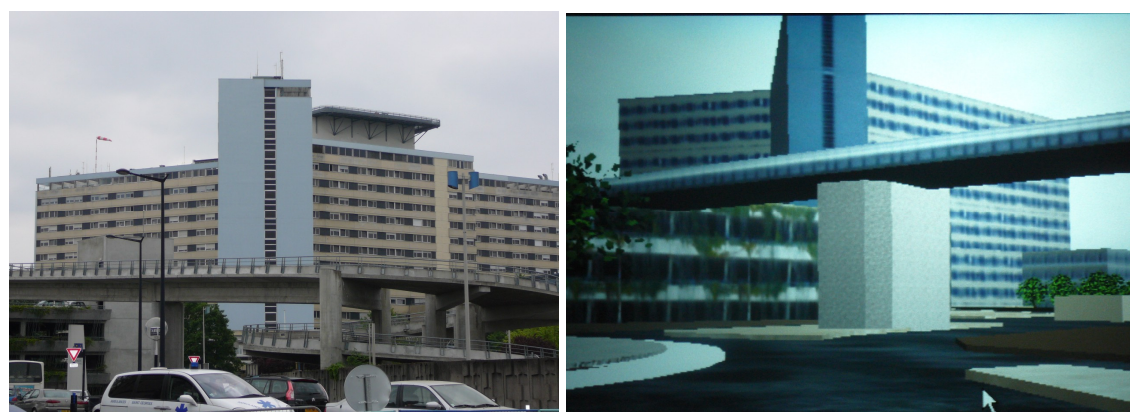

Fig. 1. Screenshots our real (left) and our virtual environment (right)

\section{Material.}

The material used in the darkened laboratory room was a DELL Precision M6300 laptop computer (RAM: 3GHz; processor: Intel Core 2 Duo T9500 2,60 Ghz) with an Nvidia Quadro FX 1600M graphics card (256Mo), a 2 x 1.88 meter screen, a projector (Optoma/ThemeScene from Texas Instrument) with rear projection. The participants were placed two meters from the display screen.

\subsection{Interface Modeling}

The Treadmill Input Device.

The two Treadmill conditions (with Automatic and Controlled Rotation) included an HP COSCOM programmable (speed, declination and acceleration) treadmill with serial Cable Ownership coupled to a Software Development Kit and an MS-EZ1 sonar telemeter. This interface enabled participants to modify the VE's visual display in real time to match his/her walking speed, with a maximum of $6 \mathrm{~km} / \mathrm{h}$. Acceleration and deceleration were applied by means of a Sonar MS-EZ1 telemeter that monitored the participant's displacements on the treadmill. The treadmill surface was divided into three parts: one for accelerating (the front of the treadmill), one for walking normally (the middle of the treadmill), and one for decelerating (the back of the treadmill). No acceleration or deceleration information was sent to the treadmill when the participant was in the walk zone. In contrast, when the participant walked into the acceleration or deceleration zone, the sonar detected length changes in the participant's position, and instructed the computer to accelerate or decelerate until the participant returned to the walk zone. Finally, the participant remaining in the deceleration zone for a prolonged period induced a stop in the environment. In the two 
Treadmill conditions, participants were able to walk, accelerate, decelerate, and stop in the VE, thus receiving physical input including optic flow, as well as kinesthetic and translational vestibular information.

For the condition Treadmill with Controlled Rotation, the participant walked on the treadmill and was informed that his/her point of view in the VE would be controlled by head rotation (providing rotational vestibular information). Head rotation movements were captured in real time by motion capture (12 OPTITRACK video-cameras, Motion point ${ }^{\mathrm{TM}}$ ). When a participant turned his/her head, the system updated the visual optic flow at a rate correlated with the head movement rotation angle (the greater the rotation angle, the faster the modification in rotational optic flow). Thus, this condition enabled translational and rotational vestibular information.

The Treadmill condition with Automatic Rotation was the same as the condition Controlled Rotation: the participant controlled its translational displacement but, on a pre-determined path; directions changes were automatically managed by the system at each intersection. The interface did not allow any rotational movement control, enabling only translational vestibular information.

\section{The Joystick Input Device.}

In both Joystick conditions (with Controlled or Automatic Rotation), displacement was controlled by a Saitek TM X52 Flight System. Forward speed, ranging from 0 to 6 $\mathrm{km} / \mathrm{h}$, was proportional to the pressure on the device, which was also used to control translational movement. Consequently, the Joystick conditions differed from the Treadmill conditions in providing optic flow, but no vestibular information.

The Joystick with Controlled Rotation condition added horizontal joystick movements, coupled to changes in rotational optic flow to simulate turning in the VE to mimic direction changes during walking. Turning speed was proportional to the magnitude of horizontal joystick movement, similar to natural head movement.

For the Joystick with Automatic Rotation, participants were informed that rotational movement was not available; turning at intersections would be automatic.

\subsection{Procedure}

Each participant completed a three-phase procedure: (1) spatial ability tests and orientation questionnaire, to assess the participant's characteristics (see below); (2) learning phase: training interface and the route-learning task under one of the four conditions; (3) restitution phase, consisting of six spatial knowledge-based tasks.

\section{Spatial Ability Tests, Orientation Questionnaire}

The GZ5 test [4] was used to measure spatial translation ability of participants; the Mental Rotation Test (MRT) [5] to measure spatial visualization and mental rotation abilities; and the Corsi's block-tapping test [6], was used to assess the visual-spatial memory span. Three self-administrated questionnaires including seven questions each (for which responses were given on a 7-point scale) were filled in by the participant. One questionnaire assessed general navigational abilities and spatial orientation in everyday life, a second evaluated the ability to take shortcuts, and the third was dedicated to the ability to use maps. 


\section{Learning Phase}

Interface Training.

Before VR exposition, each participant participated to a training phase in a different environment, to get used to interacting with one of the four interfaces that he/she will use. The training phase was finished when the participant was able to use the interface in another VE.

\section{Learning path in the VE.}

For the two conditions with Controlled Rotation, participants walked at their own speed and managed their directions in the VE. The directions at each intersection were indicated verbally by an experimenter situated behind the participant. For the two conditions with Automatic Rotation, participants mastered their speed with the Joystick or the Treadmill, but were not able to perform rotations; they were automatically managed at each intersection by the computer. Moreover, a path learning software was developed to analyze the participant's position, time, speed, collisions and interactions during the learning path. In addition, after VR exposure, the participants completed a simplified simulator sickness questionnaire (SSQ) [7] to measure the negative side effects of being immersed in graphically-rendered virtual worlds, and a questionnaire about the ergonomic of the interface used and the participant's habits.

\section{Restitution phase.}

Six tasks were performed by each participant, with a counterbalanced order.

Egocentric photograph classification task: twelve real photographs of intersections, in a random order, were presented to the participants. Participants were required to arrange the photographs in a chronological order along the path they had learned. The time limit for this task was ten minutes. The results were scored as follows: one point for a photo in the correct position, 0.5 point for each photo in a correct sequence, but not correctly placed along the path (e.g., positioning photos 4-5-6 in the right order but not placing them correctly in the overall sequence earned 1.5points). This paper-pencil task assessed the participants' ability to recall landmarks and route knowledge within an egocentric framework ([1][2][3]).

Egocentric distance estimation task: Each participant was asked to give a verbal estimate of the VR walked distance (in meters) and the figure was noted by the experimenter. This task quantified the participants' knowledge of the distances walked between the starting and ending points, which is known to be accurate when participants have acquired well-developed route knowledge [8].

Egocentric directional estimation task: This task was computer-based and consisted of presenting a series of twelve real photographs of intersections, taken from an egocentric viewpoint, in random order. Each photograph was displayed at the top of the screen, above an 8-point compass. The participant had to select the compass direction in which they were facing on the learned path when the photograph was taken. We assessed the percentage of errors and the angular error was averaged. Directional estimates are expected to be accurate when participants have acquired well-developed route knowledge [9]. 
Allocentric sketch-mapping task: Participants were required to draw a freehand sketch of the visualized route. The time limit for this task was ten minutes. One point was scored for each correct change of direction. This paper-pencil task is known to measure survey knowledge [1][2][3].

Allocentric point starting estimation task: This computer-based task consisted of presenting a series of twelve real photographs of intersections, taken from a walker's point of view, in random order. Each photograph was displayed at the top of the screen, above a 8-point compass and the participant was instructed to select the compass direction of the starting point of the learned path. We assessed the percentage errors and the mean angular errors. These direction estimates are expected to be accurate when participants have memorized a well-developed, map-like representation of the environment [10]. This task measures survey knowledge.

Real wayfinding task: This task consisted of reproducing the learned path in the real environment; this task measures the spatial transfer of participants. During this restitution task, position and time data were acquired using a Magellan ${ }^{\mathrm{TM}}$ GPS CrossOver, and a video was recorded using an AIPTEK ${ }^{\mathrm{TM}}$ DV8900 camera mounted on a bicycle helmet worn by the participant. Direction errors were calculated and expressed in percentages. When a participant made a mistake, s/he was stopped and invited to turn in the right direction. This wayfinding task is based on the use of landmarks, as well as route and survey knowledge [1][2][3], and may be considered as a naturalistic assessment of navigational abilities based. In addition, the path learning software enabled to analyze the participant's position and time data in the real environment.

\section{Participants.}

72 volunteer students participated in this experiment (36 men and 36 women). Students were randomly divided in one of the four learning conditions: 18 students were assigned to the Treadmill with Controlled Rotation condition, 18 to the Treadmill with Automatic Rotation condition, 18 in the condition Joystick with Controlled Rotation, and 18 in the Joystick with Automatic Rotation condition. All the participants had normal or corrected-to- normal vision and were native French speakers, right-handed, and had at least an A-Level or equivalent degree. Their ages were ranged from 18 to 30 years. We controlled video game experience of participants: each learning condition were composed of half gamers (who played a minimum of three hours by week during more than one year), and the other half of non video game players (who never played regularly to video games, and who were not old video gamers). The four composed learning conditions were balanced for gender and the video-gamer distribution $(\chi 2$ procedure $\mathrm{p}>.05)$. In addition, there was no significant difference in spatial abilities among the four groups, as assessed with the GZ-5 test, the Mental Rotation Test (MRT) and the Corsi's block-tapping test (respectively, $\mathrm{p}>0.180 \mathrm{p}>0.640 ; \mathrm{p}>0.200$ ). No differences were found for the orientation questionnaire $(p>0.800)$, neither for shortcuts questionnaire $(\mathrm{p}>0.600)$, or the map questionnaire $(\mathrm{p}>0.800)$. 


\section{$3 \quad$ Results}

We used a two-way ANOVA analysis [2 (Input Devices: Treadmill Vs Joystick) x 2 (Rotation: Controlled Vs Automatic)], with between-subject measures for each factor. Bravais-Pearson test was used to assess correlations.

\subsection{Learning Phase}

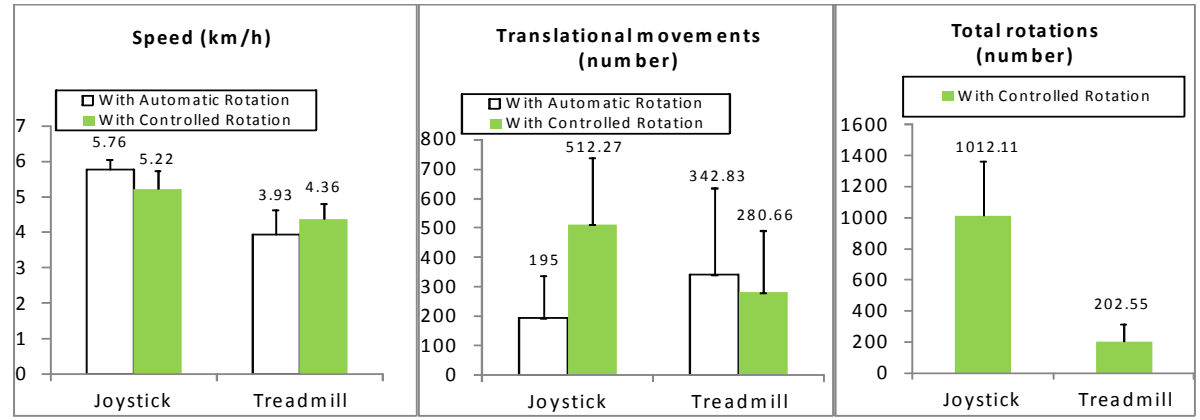

Fig. 2. Learning data according to the Input Devices (Joystick Vs Treadmill) and the Rotation (Automatic Vs Controlled)

For the Speed during learning phase, a significant effect of the Input Devices was found $\left[\mathrm{F}(1,68)=114.53 ; \mathrm{p}<0.0001 ; \eta^{2}=0.63\right]$, with higher speed during the learning phase with the Joystick compared to the Treadmill. In addition, the factor "Rotation" had no significant effect ( $>0.900)$, but the two-way interaction ("Input Devices $\mathrm{x}$ Rotation") was significant $\left[\mathrm{F}(1,68)=13.36 ; \mathrm{p}<0.001 ; \eta^{2}=0.16\right]$. With the Treadmill, speed learning was faster for with Controlled Rotation compared to the condition with Automatic Rotation. With the Joystick, the results are inversed; speed restitution was faster in Automatic Rotation condition compared to the Controlled Rotation condition.

Concerning the total translational movements (i.e, the number of accelerations/decelerations demands) during the learning path, the ANOVA analyses revealed a significant difference concerning the Rotation factor $[\mathrm{F}(1,68)=5.8 ; \mathrm{p}<0.02$; $\left.\eta^{2}=0.08\right]$. The total number of translational movements was highest in Controlled Rotation condition. An interaction "Input Devices $\mathrm{x}$ Rotation" was found $\left[\mathrm{F}(1,68)=12.84 ; \mathrm{p}<0.0001 ; \eta^{2}=0.16\right]$. With the Joystick, there were more translational movements with Controlled Rotation compared to the condition with Automatic Rotation. With the Treadmill, the results were inversed: we found more translational movements with the Automatic Rotation compared to the Controlled Rotation.

Concerning the number of rotations performed by the participant, they were summed only for the two conditions with Controlled Rotation. We used an unpaired two-tailed Student's t-test ( $\operatorname{dof}=34)$. We found a significant difference $(\mathrm{t}(9.27)$; $\mathrm{p}<0.0001 ; \eta^{2}=0.72$ ): more rotations were performed with the Joystick.

For the questionnaire about the ergonomics of the interface used, a question concerns the difficulties to perform rotations. Statistical analyses revealed a Rotation 
effect $\left[F(1,68)=7.60 ; p<0.01 ; \eta^{2}=0.10\right]$. Participants felt logically more difficulties to control their rotations in the condition With Automatic Rotation. An interaction "Input Devices x Rotation" was likewise found $\left[\mathrm{F}(1,68)=4.60 ; \mathrm{p}<0.05 ; \eta^{2}=0.06\right]$, revealing that it is only in the Treadmill with Automatic Rotation condition participants revealed rotational difficulties. In the Joystick conditions, the results were similar, whatever the possibility or not to perform rotations.

\subsection{Spatial Restitution Tasks}

For the Egocentric Photograph classification task, the ANOVA revealed no significant effect (Input Devices, p>0.600; Rotation, p>0.700; and "Input Devices x Rotation", $\mathrm{p}>0.400)$. Performances were close in all four VR learning conditions.

Concerning the Egocentric Distance Estimation Task, the ANOVA revealed a significant effect for each factor [Input Devices effect, $\mathrm{F}(1,68)=4,81 ; \mathrm{p}<0.05 ; \eta^{2}=0.07$; Rotation effect, $\left.\mathrm{F}(1,68)=12,27 ; \mathrm{p}<0.001 ; \eta^{2}=0.15\right]$, with the Joystick conditions overestimating distances compared to the Treadmill conditions, and the groups with Controlled Rotation overestimating distances compared to Automatic Rotation. The two-way interaction effect was significant $\left[\mathrm{F}(1,68)=4,44 ; \mathrm{p}<0.05 ; \eta^{2}=0.06\right]$. The distances were only overestimated in the Joystick with Controlled Rotation condition compared to the other VR conditions.

For the Egocentric Directional estimation task, the ANOVA for mean angular error revealed that the two effects taken separately were not significant (Input Devices effect, $\mathrm{p}>0.800$; Rotation effect, $\mathrm{p}>0.800$ ), but the two-way interaction was significant $\left[\mathrm{F}(1,68)=3.99 ; \mathrm{p}<0.05 ; \eta^{2}=0.06\right]$. With the Joystick, egocentric estimations were more accurate with Automatic Rotation compared to the Controlled Rotation condition, while for the Treadmill, performances were better in Controlled Rotation compared to the Automatic Rotation condition. It is to note that the results of the Joystick with Controlled Rotation and the Treadmill with Automatic Rotation conditions are very close. The results for the Joystick with Automatic Rotation and for the Treadmill with Controlled Rotation are also very close.

For the Allocentric Sketch mapping task, the ANOVA did not reveal any significant effects for the Input Devices or Rotation factors ( $p>0.800 ; p>0.300$; two-way interaction, $\mathrm{p}>0.100)$. The performances did not reveal any differences among the four VR learning conditions.

Concerning the Allocentric starting point estimation task, the only significant Input Devices effect $\left[\mathrm{F}(1,68)=4,38 ; \mathrm{p}<0.05 ; \eta^{2}=0.06\right]$ revealed by ANOVA was that the Joystick condition resulted in poorer performances than the Treadmill condition. No other effects were significant (Rotation, p>0.200; two-way interaction, p>0.800).

For the Wayfinding task (transfer task), two data (speed restitution and percentage of direction errors) were collected. For the speed restitution, the ANOVA results revealed a significant effect for Rotation $\left[\mathrm{F}(1,68)=4,22 ; \mathrm{p}<0.05 ; \eta^{2}=0.06\right]$, i.e., the group with Controlled Rotation performed better than the one with Automatic Rotation. No other difference was found (Input Devices effect, $\mathrm{p}>0.800$; two wayinteraction, $\mathrm{p}>0.900)$. For the direction error measurements, the ANOVA results revealed a significant two-way interaction $\left[F(1,68)=4.00 ; \quad p<0.05 ; \eta^{2}=0.06\right]$. 
Analysis revealed that for the Treadmill condition, performances were better with Controlled Rotation compared to the condition with Automatic Rotation. With the Joystick, the performances were more accurate with Automatic Rotation than with the Controlled Rotation condition. The best performances were performed with the Treadmill with and the Controlled Rotation. Other effects were not significant (Input Devices, $\mathrm{p}>0.800$; Rotation, $\mathrm{p}>0.300$ ).

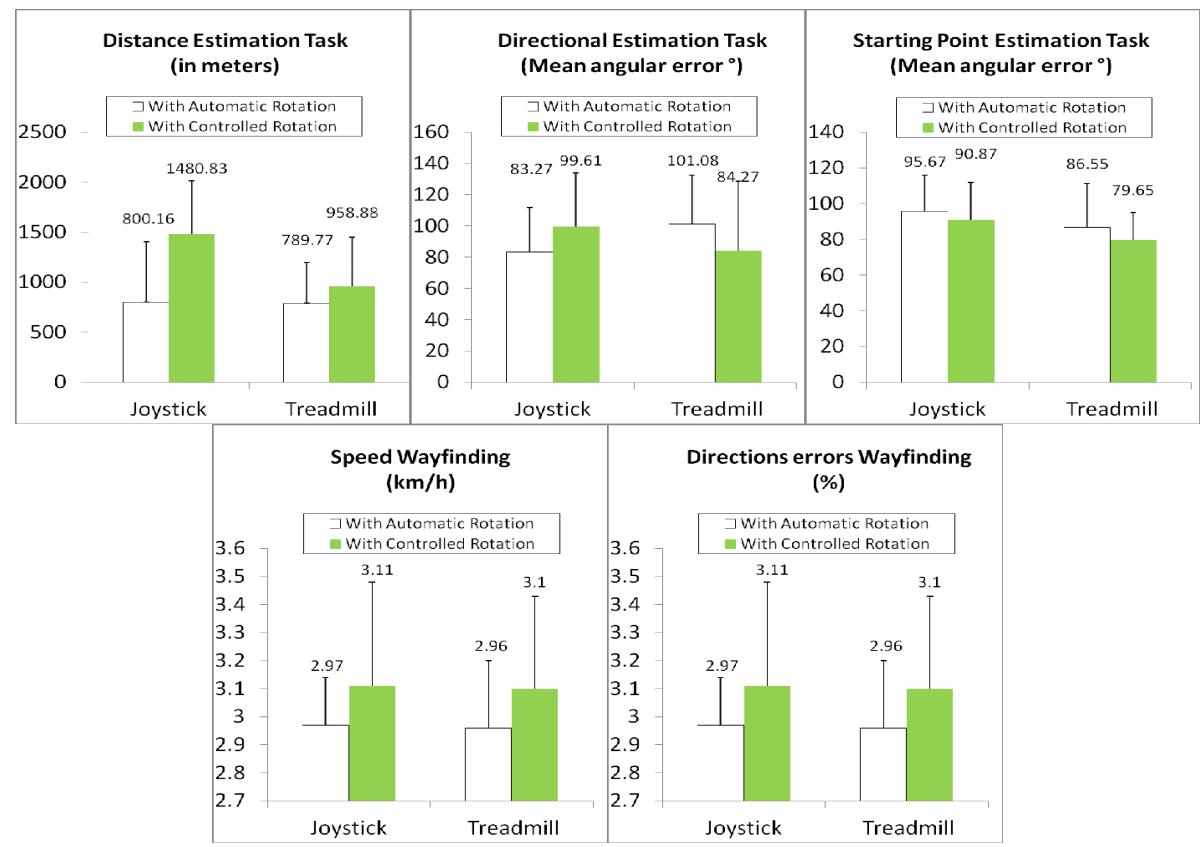

Fig. 3. Significant results for our spatial restitution tasks (Input Devices Vs. Rotation)

\subsection{Correlations}

We present only the principal correlations between data learning (translational movements, performed rotations), paper pencils tasks, the three orientations questionnaires, and the six spatial restitution tasks. No correlations were found about spatial pencil papers tasks and spatial restitution tasks. Concerning the orientation questionnaires, we found a negative correlation between the questionnaire assessing the abilities to use maps and the starting point estimation task $(\mathrm{p}=0.02, \mathrm{r}=-0.37)$. No correlations were found concerning the other spatial restitution tasks. For the data acquired during the VE learning path, a positive correlation was found between the time to finish the learning path and the sketch mapping task $(\mathrm{p}=0.04, \mathrm{r}=0.34)$. No correlations were found between rotations, translational movements and spatial restitution tasks. 


\section{Discussion}

To recall, the goal of this study is to understand the impact on spatial transfer, of two Input Devices with two different levels of motor activities (the manipulation of a Joystick Vs a Treadmill) and the possibility or not to control rotations. In manipulating these two factors, we are forced to manipulate body-based information. More precisely, whatever the type of Rotation (i.e., Automatic Vs Controlled), the Joystick enables visual information, but no vestibular information (no head displacement). The only difference was, in the condition Joystick with Controlled Rotation, participant was able to master their translational displacements and their directions to explore freely the VE, while in the Joystick with Automatic Rotation condition, participant followed a predetermined path, and was able to control only their speed displacement; the rotations being controlled by computer at each intersection. The Treadmill with Controlled Rotation condition enabled to perform rotations (rotational head movements) during the learning path, providing translational and rotational vestibular information. In the Treadmill with Automatic Rotation condition, head rotations were blocked and translational vestibular information were activated. As provided in Joystick with Automatic Rotation, participant was able to control his/her displacement speed, and directions changes at each intersection were managed by the computer. We present our results according to the egocentric, allocentric and transfer tasks used.

\subsection{Egocentric Tasks}

For the Photograph classification task, whatever the Rotation or the Input Devices factors used, no statistical differences were found for this task. To recall, this task consisted in ordering chronologically twelve photos of the real environment, with an egocentric point of view. So, the motor activity and the possibility or not to perform rotations of our four interfaces seems to have little impact on this task. These results are in accordance with literature. For example, Wallet et al.[2] found, on the same type of task, that visual fidelity of a VE was more important than the interface used. We may wonder that different body-based information and attentional levels provided by our four interfaces had a little impact on tasks that do not require the recall of an action. It could mean also that the visual fidelity of our VE was perceived in the same way by the participants, whatever the interfaces used. If some differences appear they cannot be explained by this factor.

Concerning the Egocentric Distance Estimation Task (which consisted in evaluating the total distance travelled during the learning path phase), the results showed a significant difference for Input Devices, in favor of the Treadmill (vestibular information present) compared to the Joystick (i.e., no vestibular information because no head movements). These results are in accordance with different authors [11][12] who demonstrated that vestibular information is important to correctly estimate distances. An effect Rotation was also found, showing a distance overestimation for the conditions with Controlled Rotation compared to the conditions with Automatic Rotation. Statistical analyses revealed an interaction "Input Devices x Rotation". The distance estimates were very close for the Treadmill with Controlled or Automatic Rotation 
conditions, and the Joystick with Automatic Rotation. An overestimation for the Joystick with Controlled Rotation condition was found, explaining the Rotation effect described above. Finally, the Rotation (and rotational vestibular information), had no impact for the Treadmill. These results are coherent with [14] where the importance of the translational vestibular information on distance estimation is confirmed, and where no effect of rotational vestibular information was found. In contrast, in the two Joystick conditions (only visual information provided), we can see an overestimation only with the Controlled Rotation condition. For several authors, visual information would be sufficient to estimate distance [15], explaining maybe the results with $A u$ tomatic Rotation. These results are new and difficult to explain. This may be due to the fact that two directions and the visuomotor coordination requested could interfere on visual and cognitive processes. Maybe the visuomotor coordination of the joystick was higher for gamers(compared to no gamers). It would be interesting to add a condition comparing video game experience to improve our conclusions. It is important to note that in a walking activity, the rotation seems not to be important for distance estimation. But, with a Joystick, the Controlled Rotation affects the distance estimation.

For the Egocentric Directional Estimation task (which consisted for participant to indicate the direction he took, according to real photographs of intersections), results showed an interaction "Input Devices x Rotation". With the Joystick, the condition with Automatic Rotation gave the best performances, while with the Treadmill, contrary to the previous task, the best performances were with the Controlled Rotation condition (and rotational vestibular information). Finally, according to the motor activity of the interface used, the Rotation factor had a different impact. Our results corroborate the results found by other authors [12] [13] showing that 1) vestibular information improves egocentric representations 2) rotational vestibular information, rotational head movements allow increasing egocentric and perception-action representations. However, for the Joystick, the Controlled Rotation decreases once again egocentric perceptions. Moreover, statistical analyses showed that in the Joystick conditions, translational movements strongly increased with the Controlled Rotation (compared to the Automatic Rotation condition). For the two Treadmill conditions, the number of translational movements was quite similar whatever the type of Rotation used. When we compared the number of rotations (Joystick and Treadmill with Controlled Rotation conditions), statistical analyses revealed almost five times more interactions for the Joystick than for the Treadmill. These results concerning the number of interactions seem to support our assumptions about the visuomotor difficulties to control two directions with the joystick. Moreover, to the question of the difficulties to perform rotations, statistical analyses showed a significant effect of Rotation, where the group with Automatic Rotation had more difficulties to improve rotations compared to the Controlled Rotation group. These results seem to be logical because in the Automatic Rotation condition, participants were not able to control their rotations. An interaction "Input Devices x Rotation" also appeared. Surprisingly, this result showed that the rotation difficulties felt by participants concerned only the condition Treadmill with Automatic Rotation. In other words, when the interaction is natural, as a walking activity, the rotation seems to be necessary for a $3 \mathrm{D}$ navigational 
task. On the other hands, with the Joystick, the results to this question were very close, with Automatic or Controlled Rotation. Thus, participants did not felt the need to improve their rotations with the two-Joystick conditions. With a Joystick, it seems that the Controlled Rotation is not always necessary, confirming the great participants performances in the Joystick with Automatic Rotation condition. These results can be interpreted in different ways: 1) a walking activity is more natural than a joystick, explaining the similar number of interactions With Automatic or Controlled Rotation. But the Controlled Rotation and rotational vestibular information improves the egocentric representations 2) it seems to indicate that the two directions manipulated with the joystick is difficult. Maybe the attentional levels of participants are divided between the control of the joystick and the visual perception of the VE. Participants could have visuomotor coordination hand difficulties [16]. Moreover, unlike with the Treadmill, participants did not interact in the same manner with the Joystick and the Automatic or the Controlled Rotation. 3) Maybe the Controlled Rotation of the Joystick took in account to many rotational movements of the hand. Adding a condition where the Controlled Rotation of the Joystick took into consideration fewer rotations should give new information about our results. It is to note that no correlations were found between translational movements/rotations and our six spatial restitution tasks.

To summarize:

- The distance perception is optimized in a walking activity (whatever the Rotation factor). A joystick with Automatic Rotation permits also to assess correctly distances (only with visual information).

- The use of a Joystick and a Controlled Rotation decreases egocentric representations. With a joystick, only the control of translational movements seems to be sufficient to acquire egocentric spatial knowledge, similarly to a walking interaction close to the real life (i.e., Treadmill with Controlled Rotation).

- In a walking activity, the rotation, rotational head movements optimize the creation of egocentric representations. The absence of rotational vestibular information with a Treadmill affects negatively spatial egocentric representations.

\subsection{Allocentric Tasks}

Concerning the allocentric sketch mapping task, no significant differences were found. We can still observe a positive correlation between this task and the time to learn the path. The higher the completion time, the better the performances. These results support the L-R-S model of Siegel and White [9], who admitted survey representations to be improved with a long and repeated exploration of the VE.

For the Allocentric Starting-point estimation task, the results indicated better performances with the Treadmill compared to the Joystick, whatever the Rotation Factor. A walking activity, natural and transparent could optimize allocentric representations. Due to the significant effects absence of the Rotation factor with the Treadmill, we can suppose that for increasing allocentric representations, translational vestibular information is more important than rotational vestibular information. These results are consistent with the findings of [14][17], regarding the importance of walking activity in the development of allocentric representations. However, these results could be 
different with a joystick condition with gamers participants, accustomed to use a joystick. Once again, it seems to be interesting to add this condition as a factor. Moreover, a positive correlation between the questionnaire assessing the abilities to use maps and this allocentric task can be observed. It means the allocentric representations would be strongly linked with allocentric participants experience and cognitive processes used to manipulate allocentric representations [3].

Due to the different results on the allocentric tasks, it is difficult to summarize this part. In one case (the sketch-mapping task), we did not find motor activity effect. It is already admitted the allocentric representations are related to different cognitive processes and to the manipulation and the repetition of spatial representations [9]. On the other hand (allocentric starting-point estimation task), we found a great impact of the walking activity on allocentric representations, meaning a walking activity improved the creation of allocentric representations [14]. A hypothesis concerns the allocentric tasks used. Indeed, different authors argue that drawing could be an ability to sketch correctly a route [18]. Maybe these two allocentric tasks did not assess the same cognitive processes or spatial representations. The debate about the impact of motor activity on allocentric representations still exists. However, due to the absence of Rotation effect, we can state that the rotational component seems to be negligible for tasks mainly driven by allocentric spatial representations [20].

\subsection{Spatial Transfer (The wayfinding task)}

To recall, this task consisted in reproducing in the real environment, the learned path. We collected two data: the mean speed to finish the task and the directions errors percentage. For the mean speed, the statistical analyses showed a Rotation effect; the mean speed was higher for the conditions with Controlled Rotation, compared to the condition with Automatic Rotation, whatever the Input Devices used. It can be supposed that the free learning exploration of the VE at each intersection (whatever the Input Devices used) is close to a real learning, optimizing the speed transfer in the real environment. For the percentage of direction errors, we observed an interaction "Input Devices x Rotation". With the Treadmill, performances are the best with Controlled Rotation, while with the Joystick, best performances have been observed with Automatic Rotation. The Treadmill with Controlled Rotation condition allows the participants to optimize performances in term of speed and errors percentage. Grant and Magee [19], in a spatial transfer task, already found these results, in comparing a walking interface and a joystick, but both with Controlled Rotation. Nevertheless, the Rotation factor was not controlled in their study, the superiority of walking interface over joystick may have been induced by freedom of rotation rather than the physical engagement provided by the walking interface, as demonstrated in our study. We can also suppose that the Treadmill with Controlled Rotation is very close to a real walking activity, and optimize the spatial transfer performances. However, once again, the Controlled Rotation negatively impacts spatial performances with the Joystick. These results are very similar to the Egocentric Estimation Task, and the Rotation factor generates different impact according to the Input Devices and the motor activity provided: in a walking situation, a Controlled Rotation (with rotational vestibular 
information) increases performances, while with a Joystick, a Controlled Rotation affect negatively spatial acquisition. As for the Egocentric Estimation Task, we suppose performances in the Joystick with Controlled Rotation could be due to the difficulties to manage two directions with the hand, generating visuomotor problems [16]. Considering a similar joystick condition with game experience could give more information about spatial transfer.

To summarize:

- Translational and rotational vestibular information provided by Controlled Rotation with the Treadmill optimize spatial transfer [19]. Only translational vestibular information decreases spatial transfer performances.

- With the Joystick, the Automatic Rotation enabled the best performances.

\section{Conclusion}

According to our experiments, the motor activity during an interaction and the manual control of rotations had different impacts on spatial transfer. Translational and rotational vestibular information provided by the Treadmill with Controlled Rotation optimizes spatial egocentric and transfer performances, as well as the Joystick with Automatic Rotation. The question concerning the allocentric representations is most contrasted. In one case a walking activity enhances performances (starting-point estimation task), while in another, no differences were found (sketch mapping task). Further investigation is required to clarify this point.

The novelty of this research concerns the bad performances, whatever the tasks performed, with the Joystick and the Controlled Rotation, though often used in videogames or on spatial cognition research. The Joystick Input Device may offer an advantage for spatial learning under specific conditions (translational control), close to the Treadmill with Controlled Rotation, but not others (translational and rotational controls). All our results showed better performances in the Joystick with Automatic Rotation condition (close to the Controlled Rotation Treadmill) compared to the Automatic Rotation Joystick condition. One hypothesis if that vertical and horizontal hand movements do not provide adequate metaphors of translational and rotational displacements to implement a dialogue between the cognitive and sensorimotor systems that contribute to spatial learning. A condition where participants can only manage the direction of their displacement would give some information about the visuomotor coordination of two directions with the hand. This challenges the debate on the possible advantage of active navigation with a joystick (compared to simple observation), where some studies detected a benefit for spatial learning performances $[21][1][2][8])$ but others did not ([22][23][24]). Moreover, joystick interfaces are more widely used than treadmills, since they are less expensive, easier to implement from a technological standpoint. This device is also often adapted to the user's needs, notably for people with mobility issues. This the case with the elderly, patients with Parkinson's or Alzheimer's diseases, or sensorimotor injuries ([25][26][27]). Thus, clarifying the impact of joystick use represents a research challenge and is essential to resolve fundamental issues for clinical neuropsychological applications. 


\section{References}

1. Wallet, G., Sauzeon, H., Rodrigues, J., Larrue, F., N'Kaoua, B.: Virtual / Real Transfer of Spatial Learning: Impact of Activity According to the Retention Delay. St. Heal. (2010)

2. Wallet, G., Sauzeon, H., Pala, P.A., Larrue, F., Zheng, X., N'Kaoua, B.: Virtual/Real transfer of spatial knowledge: benefit from visual fidelity provided in a virtual environment and impact of active navigation. Cyberpsychology, Behavior and Social Networking 14(7-8), 417-423 (2011)

3. Larrue, F., Sauzeon, H., Aguilova, L., Lotte, F., Hachet, M., N'Kaoua, B.: Brain Computer Interface Vs Walking Interface in VR: The impact of motor activity on spatial transfer. In: Proceedings of the 18th ACM Symposium on Virtual Reality Software and Technology (VRST 2012), pp. 113-120. ACM, New York (2012)

4. Guilford, J.P., Zimmerman, W.S.: The Guilford-Zimmerman Aptitude Survey. Journal of Applied Psychology (1948)

5. Vandenberg, S.G., Kuse, A.R.: Mental rotations, a group test of three-dimensional spatial visualization. Perceptual and Motor Skills 47(2), 599-604 (1978)

6. Wechsler, D.: Manual for the Wechsler Adult Intelligence Scale-Revised. Psychological Corporation, New York (1981)

7. Kennedy, R., Lane, N., Berbaum, K., Lilienthal, M.: Simulator Sickness Questionnaire: An Enhanced Method for Quantifying Simulator Sickness. The International Journal of Aviation Psychology 3(3), 203-220 (1993)

8. Waller, D., Hunt, E., Knapp, D.: The Transfer of Spatial Knowledge in Virtual Environment Training. Presence: Teleoperators and Virtual Environments 7(2), 129-143 (1998)

9. Siegel, A.W., White, S.H.: The development of spatial representations of large-scale environments. Advances in Child Development and Behavior 10, 9-55 (1975)

10. Ruddle, R.A., Volkova, E., Mohler, B., Bülthoff, H.H.: The effect of landmark and bodybased sensory information on route knowledge. Memory \& Cognition 39(4) (2011)

11. Waller, D., Richardson, A.R.: Correcting distance estimates by interacting with immersive virtual environments: effects of task and available sensory information. Journal of Experimental Psychology: Applied 14(1), 61-72 (2008)

12. Klatzky, R.L., Loomis, J.M., Beall, A.C., Chance, S.S., Golledge, R.G.: Spatial Updating of Self-Position and Orientation During Real, Imagined, and Virtual Locomotion. Psychological Science 9(4), 293-298 (1998)

13. Chance, S.S., Gaunet, F., Beall, A.C., Loomis, J.M.: Locomotion Mode Affects the Updating of Objects Encountered During Travel: The Contribution of Vestibular and Proprioceptive Inputs to Path Integration. Presence: Teleoperators and Virtual Environments 7(2), 168-178 (1998)

14. Ruddle, R.A., Volkova, E., Bülthoff, H.H.: Walking improves your cognitive map in environments that are large-scale and large in extent. ACM Transactions on Computer-Human Interaction 18(2), 1-20 (2011)

15. Riecke, B.E., Cunningham, D.W., Bülthoff, H.H.: Spatial updating in virtual reality: the sufficiency of visual information. Psychol. Res. 71(3), 298-313 (2007)

16. Richardson, A.E., Powers, M.E., Bousquet, L.G.: Video game experience predicts virtual, but not real navigation performance. Computers in Human Behavior 27(1) (2011)

17. Ruddle, R.A., Lessels, S.: The benefits of using a walking interface to navigate virtual environments. ACM Transactions on Computer-Human Interaction 16(1), 1-18 (2009)

18. Golledge, R.G.: Human wayfinding and cognitive maps. In: Wayfinding Behavior, pp. 5-45. The John Hopkins University Press, Baltimore (1999) 
19. Grant, S.C., Magee, L.E.: Contributions of Proprioception to Navigation in Virtual Environments. Human Factors: The Journal of the Human Factors and Ergonomics Society 40(3), 489-497 (1998)

20. Ruddle, R.A., Péruch, P.: Effects of proprioceptive feedback and environmental characteristics on spatial learning in virtual environments. International Journal of HumanComputer Studies 60(3), 299-306 (2004)

21. Péruch, P., Vercher, J., Gauthier, G.M.: Acquisition of Spatial Knowledge Through Visual Exploration of Simulated Environments. Ecological Psychology 7(1), 1 (1995)

22. Wilson, P., Foreman, N., Gillett, R., Stanton, D.: Active Versus Passive Processing of Spatial Information in a Computer-Simulated Environment. Ecological Psychology (1997)

23. Wilson, P.N.: Active Exploration of a Virtual Environment Does Not Promote Orientation or Memory for Objects. Environment and Behavior 31(6), 752-763 (1999)

24. Wilson, P.N., Péruch, P.: The influence of interactivity and attention on spatial learning in a desktop virtual environment. Current Psychology of Cognition 21, 601-633 (2002)

25. Rizzo, A., Schultheis, M., Kerns, K., Mateer, C.: Analysis of assets for virtual reality applications in neuropsychology. Neuropsychological Rehabilitation 14(1), 207-239 (2004)

26. Skelton, R.W., Bukach, C.M., Laurance, H.E., Thomas, K.G., Jacobs, J.W.: Humans with traumatic brain injuries show place-learning deficits in computer-generated virtual space. Journal of Clinical and Experimental Neuropsychology 22(2), 157-175 (2000)

27. Lloyd, J., Powell, T.E., Smith, J., Persaud, N.V.: Use of a virtual-reality town for examining route-memory, and techniques for its rehabilitation in people with acquired brain injury. In: Proceedings of the 6th International Disability, Virtual Reality and Associated Technologies, Esbjerg, pp. 175-182 (2006)

28. Montello, D.R.: Navigation. In: Shah, P., Miyake, A. (eds.) The Cambridge Handbook of Visuospatial Thinking, pp. 257-294. Cambridge University, Cambridge (2005)

29. Ishikawa, T., Montello, D.R.: Spatial knowledge acquisition from direct experience in the environment: individual differences in the development of metric knowledge and the integration of separately learned places. Cognitive Psychology 52(2), 93-129 (2006) 